\title{
Noninvasive diagnosis of compensated cirrhosis using an analysis of the time-intensity curve portal vein slope gradient on contrast-enhanced ultrasonography
}

\author{
Yuichi Goto $\cdot$ Koji Okuda • Gen Akasu • \\ Hisafumi Kinoshita $\cdot$ Hiroyuki Tanaka
}

Received: 25 November 2011/Accepted: 7 August 2013/Published online: 18 October 2013

(C) The Author(s) 2013. This article is published with open access at Springerlink.com

\begin{abstract}
Purpose We measured the slope gradients (SGs) of the vascular time-intensity curves (TICs) of the intrahepatic vessels on contrast-enhanced ultrasonography (CEUS). The aim of this study was to assess the diagnostic accuracy of the SG of each hepatic vessel, particularly the portal vein (PV), for detecting cirrhosis and to compare this method with conventional modalities.

Methods Fifty-one preoperative patients underwent CEUS, and the TICs were plotted. The SGs of the hepatic artery, PV and hepatic vein were obtained from the linear functions between the slope of the arrival time of the contrast agent and the peak enhancement time of each vessel. The transit times and levels of biochemical markers were also measured. The patients were divided into three groups according to the Metavir score: F0/1 group $(n=14), \mathrm{F} 2 / 3$ group $(n=21)$ and F4 group $(n=16)$.

Results The PVSG significantly decreased in the F4 group (F0/1: $29.1 \pm 2.27, \quad \mathrm{~F} 2 / 3: \quad 23.1 \pm 1.86, \quad \mathrm{~F} 4$ : $14.7 \pm 2.13$ ). The PVSG demonstrated high accuracy for diagnosing cirrhosis and was correlated with the levels of ICG-R15 and hyaluronic acid (Spearman rank correlation; $\rho=-0.5691, p<0.001$ and $\rho=-0.4652, p=0.0006$ ). Conclusions The PVSG has the potential to be a diagnostic marker for identifying patients with well-compensated cirrhosis.
\end{abstract}

Keywords Diagnosis - Compensated cirrhosis ·

Contrast-enhanced ultrasonography .

Time-intensity curve $\cdot$ Portal vein

Y. Goto $(\bowtie) \cdot$ K. Okuda · G. Akasu · H. Kinoshita · H. Tanaka Division of Hepatobiliary and Pancreatic Surgery,

Department of Surgery, Kurume University,

67 Asahi-machi, Kurume, Fukuoka 8300011, Japan

e-mail: gotou_yuuichi@med.kurume-u.ac.jp

\section{Introduction}

Liver cirrhosis is a chronic, diffuse and progressive condition characterized by the development of fibrosis and the conversion of the normal liver architecture into structurally abnormal nodules. Although more than $1 \%$ of some populations have histological evidence of cirrhosis, cases of compensated cirrhosis often go clinically undetected for prolonged periods of time [1]. In patients with chronic liver disease, the presence of cirrhosis and the degree of fibrosis are important factors, as they help to determine therapeutic options and can direct patient management, particularly in cases in which hepatic resection is indicated for concomitant primary malignancy. Several noninvasive evaluations of chronic liver disease have been reported to be useful [2-5]; however, well-compensated cirrhosis patients may have normal or near-normal levels of markers; thus, these parameters are not effective for evaluating the degree of liver disease, which is critical for predicting perioperative risks. Although a liver biopsy is considered the gold standard for assessing the severity of fibrosis and the presence of cirrhosis, the fact that only one part of the liver is sampled leads to false-negative results in up to $30 \%$ of cases $[6,7]$. Furthermore, biopsies are not without inherent risks and cannot be performed repeatedly in follow-up. Therefore, there is a need for a simple, reliable and noninvasive technique for assessing hepatic fibrosis and cirrhosis.

Studies have shown that contrast-enhanced ultrasonography (US) exhibits high accuracy in the diagnosis of cirrhosis [8-10]. The time of onset of US contrast enhancement of the hepatic veins (hepatic vein arrival time: HVAT) is reported to be especially useful. A reduced HVAT is correlated with an increased severity of liver disease due to arteriovenous shunting and arterialization of 
the capillary beds in the liver. A recent study demonstrated that measuring the HV-HA interval time and $\mathrm{HV}-\mathrm{PV}$ interval time, which corresponds to the interval from the arrival time of the contrast agent into the hepatic artery (HA) or portal vein (PV) to the hepatic vein (HV), can be used to differentiate mild fibrosis from more severe degrees of fibrosis in patients with chronic liver disease [11, 12]. However, the HVAT is influenced by intrahepatic circulatory changes rather than extrahepatic hemodynamic changes, which are also important for assessing the severity of liver disease [13].

Liver cirrhosis is characterized not only by changes in the intrahepatic circulation, but also by extrahepatic hemodynamic changes, such as portocaval and gastrointestinal shunting, splenic circulatory changes and hypersplenism. These changes affect the inflow hemodynamics of the PV as well as the HA as a result of the "hepatic arterial buffer response" [14]. Based on this background, we measured the slope gradient (SG) of the intrahepatic vascular intensity curve using a contrast agent, Sonazoid (GE Healthcare, Oslo, Norway), focusing on the PV $[15,16]$.

The aim of this prospective study was to assess the diagnostic accuracy of the SG of each hepatic vessel, particularly of PV, for detecting and characterizing the severity of compensated cirrhosis compared with conventional biochemical modalities. We also assessed the advantages of evaluating the SG compared with recently reported transit time analyses using a contrast agent to determine the HVAT, HV-HA interval time and HV-PV interval time in diagnosing compensated cirrhosis in patients with liver tumors.

\section{Materials and methods}

\section{Patients}

Fifty-one preoperative patients who were referred to our Department of Surgery between May 2009 and February 2010 were enrolled in this study. All patients had liver tumor(s) and were scheduled to undergo hepatic resection or ablation therapy. Patients were excluded if they had (a) a previous history of hepatobiliary-pancreatic surgery, splenectomy, portocaval shunt surgery or TIPS, (b) liver tumor(s) measuring more than $5 \mathrm{~cm}$ in size or located adjacent to the major portal or hepatic veins (this would affect the hepatic circulation) or (c) chronic renal disease, cardiac dysfunction or chronic obstructive pulmonary disease (all of which induce systemic hemodynamic abnormalities).

The characteristics of the patients were as follows: there were 35 males and 16 females with a mean age of 67.03 years (range 43-88 years). Twenty-eight patients were $\mathrm{HCV}$ antibody-positive, three patients were $\mathrm{HBV}$ surface antigen-positive, one patient had $\mathrm{HBV}$ and $\mathrm{HCV}$ coinfection, four patients had alcoholic hepatitis and two patients had cryptogenic hepatitis. All patients were classified as having a Child-Pugh grade A status. The mean size of the tumors was $24.16 \pm 8.70 \mathrm{~mm}$, and the mean number of tumors per patient was $1.27 \pm 0.45$. Informed consent to participate in this study was obtained from all patients.

\section{Ultrasound examinations}

All patients were tested in the morning after an overnight fast. One surgeon with over 6 years of experience in US, including Doppler US, and over 3 years of experience with contrast-enhanced US who was blind to the clinical data performed all tests. The ultrasound scanner was a Toshiba Aplio XG (Toshiba, Tokyo, Japan) with a curved 3.75 MHz transducer. The apparatus settings for the low mechanical index (MI) harmonic imaging were standardized as follows: gain of 80 , dynamic range of $50 \mathrm{~dB}, \mathrm{MI}$ of 0.21 , with the focus point $8 \mathrm{~cm}$ from the surface. In each case, the right hepatic artery (HA), right portal vein (PV) and right hepatic vein (HV) were simultaneously scanned using the right intercostal view. The microbubble contrast agent was Sonazoid (GE Healthcare, Oslo, Norway). A 23-G cannula was inserted into the left antecubital fossa vein of the patient. Sonazoid was injected manually at a dose of $0.0075 \mathrm{ml} / \mathrm{kg}$, followed by a rapid normal saline flush $(10 \mathrm{ml})$. Following injection of Sonazoid, the patient was asked to hold their breath for as long as possible (at least $30 \mathrm{~s}$ ), and gray scale cine images were digitally recorded onto the hard disk drive of the US scanner.

Data analysis

The brightness value and time analyses were performed using an off-line personal computer with the Clip Washer (Toshiba, Tokyo, Japan) and ImageJ (NIH) software programs, which are available free of charge for multiple operating systems at http://rsb.info.nih.gov/ij/. First, we decompressed the cine images saved in the Audio Video Interleave (AVI) format into uncompressed AVI files. In the uncompressed AVI file, the interval of each frame was 1/15 of a second. A total of 15 frames of the gray scale images were processed per second using the ImageJ software program. We observed the cine image frame-by-frame, and the arrival time of each vessel was set at the time of the first echogenic microbubble observed in the vessel.

We set circular ROIs in the HA, PV and HV and measured the brightness values automatically using the ImageJ software program (Fig. 1). The brightness value of each pixel was expressed as 0 at minimum and 255 at maximum. A brightness level in the ROI of 255 signifies that all pixels 
in the ROI are completely filled with pixels with a 255 brightness value, which means that the established circular ROI is visually filled with contrast agent. After measuring the

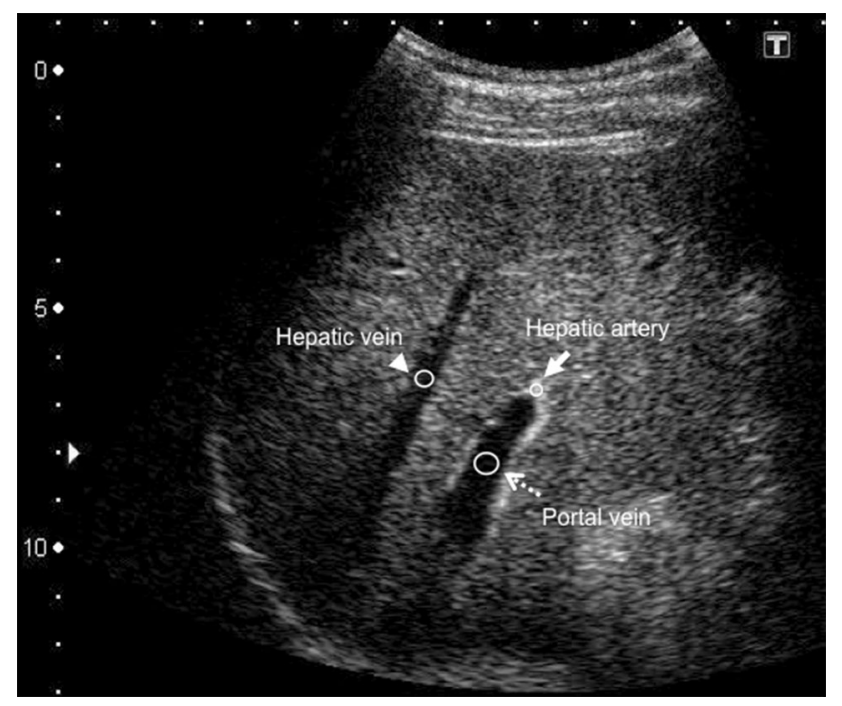

Fig. 1 The intensity of each vessel was measured by setting circular ROIs in each vessel using the ImageJ software program. The arrow is the ROI for the HA, the broken arrow is the ROI for the PV and the arrowhead is the ROI for the HV. The ROIs were set in the vessels at a depth of $6-10 \mathrm{~cm}( \pm 2 \mathrm{~cm}$ from the focus point) from the surface brightness values in each vessel, we created time-intensity curves of the three vessels using the Excel software program (Microsoft, WA, USA) (Fig. 2). The peak enhancement time was evaluated according to the time-intensity curve (TIC). We then calculated the gradient of the slope between the arrival time and the peak enhancement time as a linear function according to the linear approximation method using Excel. We named the gradient of the obtained linear function the slope gradient (SG) (Fig. 3).

Histological assessment of the specimens

In 33 patients, a histological assessment of fibrosis was performed using the resected specimen obtained at the time of surgery for the liver tumor. In 18 patients, the histological assessment was performed using an intraoperative biopsy with a 17-gauge needle at the time of surgical ablation. In all patients, the histological findings were interpreted by two independent pathologists who were blinded to the findings of contrast-enhanced US and the other clinical data. The stage of fibrosis was evaluated semiquantitatively using the Metavir scoring system [17], as follows: F0 = no fibrosis, F1 = portal fibrosis without septa, F2 = portal fibrosis and few septa, F3 = numerous septa without cirrhosis and F4 $=$ cirrhosis. The fibrosis stages in all patients
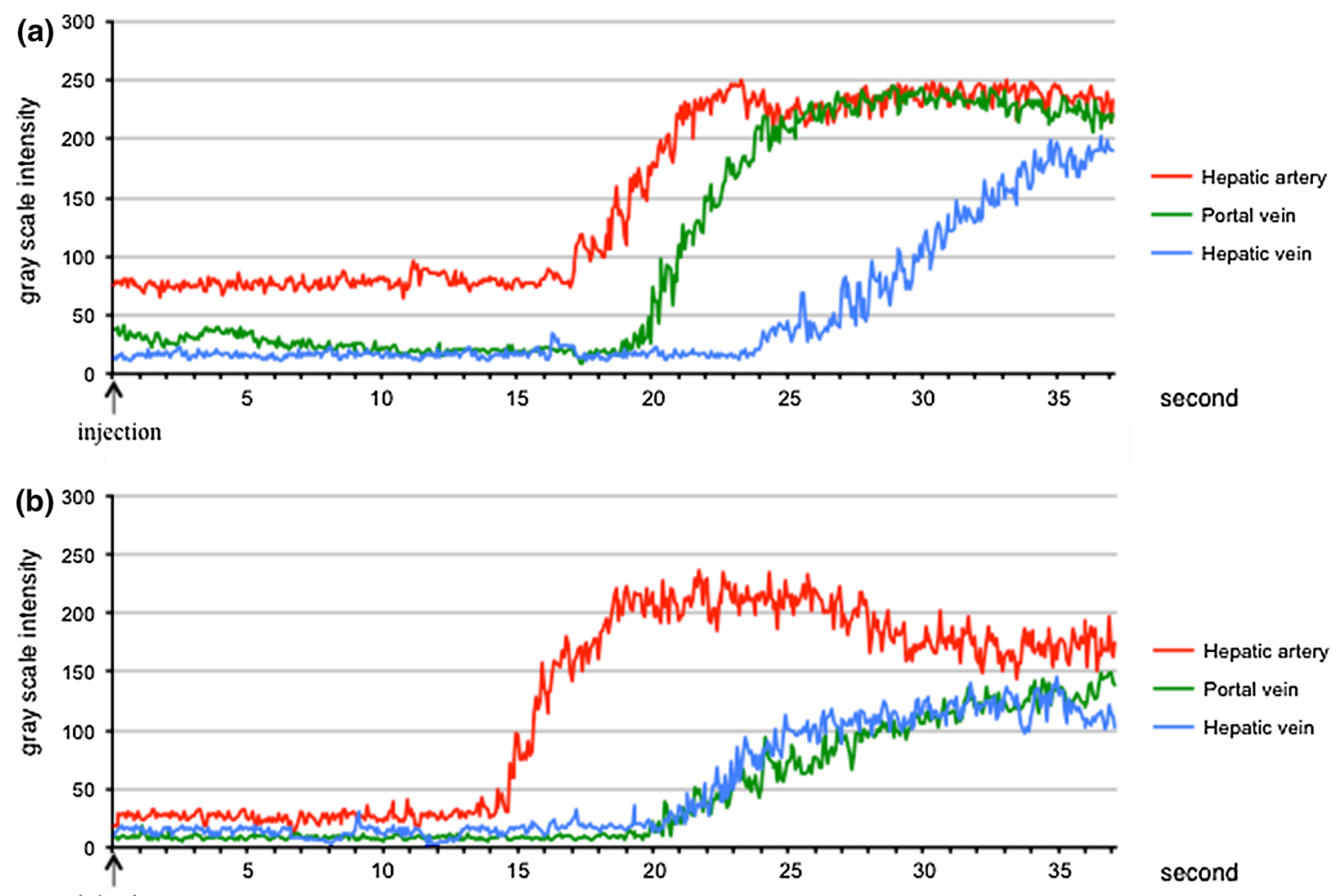

Fig. 2 Time-intensity curves of each vessel in a normal liver (a) and a cirrhotic liver (b). The red line is the signal intensity of the HA, the green line is the signal intensity of the PV and the blue line is the signal intensity of the HV. In the patients with cirrhosis, the slope of the PV is gentle compared with that observed in the patients with a normal liver 
Fig. 3 An example of the slope gradient of the portal vein (PV). The PV slope gradient (PVSG) was obtained according to the linear approximation method between the arrival time (a) and the peak enhancement time (b). In the figure, the PVSG is 16.7

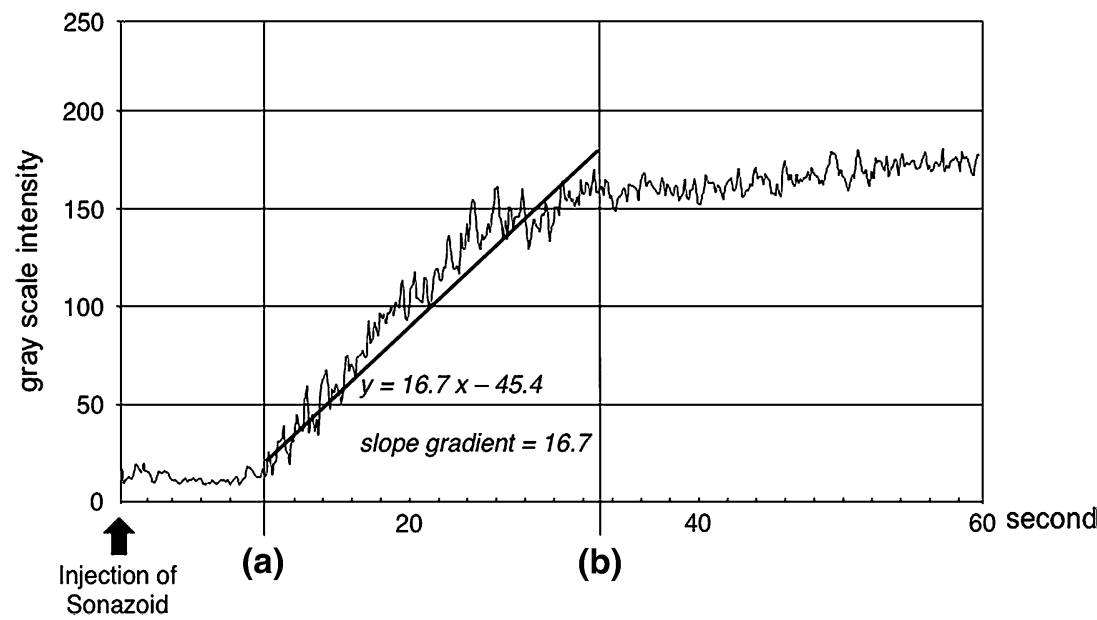

Table 1 Characteristics of the patients and liver tumors in each group

\begin{tabular}{llll}
\hline & $\begin{array}{l}\text { F0/1 group } \\
n=14\end{array}$ & $\begin{array}{l}\text { F2/3 group } \\
n=21\end{array}$ & $\begin{array}{l}\text { F4 group } \\
n=16\end{array}$ \\
\hline Age (years) & $69.6 \pm 2.97$ & $65.0 \pm 2.42$ & $67.4 \pm 2.77$ \\
Male/female & $10 / 4$ & $15 / 6$ & $10 / 6$ \\
Body mass index $\left(\mathrm{kg} / \mathrm{m}^{2}\right)$ & $21.9 \pm 0.62$ & $22.2 \pm 0.51$ & $22.7 \pm 0.58$ \\
HCV/HBV/HCV+HBV/ & $1 / 0 / 0 / 0 / 0$ & $14 / 2 / 1 / 2 / 2$ & $13 / 1 / 0 / 2 / 0$ \\
$\quad$ alcoholic/cryptogenic & & & \\
Child-Pugh grade A/B & & & $16 / 0$ \\
Tumor size (mm) & $24.5 \pm 2.20$ & $27.3 \pm 1.80$ & $19.7 \pm 2.06$ \\
Tumor number & $1.29 \pm 0.12$ & $1.24 \pm 0.10$ & $1.31 \pm 0.11$ \\
AST (IU/L) & $24.6 \pm 5.33$ & $50.4 \pm 4.35$ & $58.3 \pm 4.99$ \\
ALT (IU/L) & $18.7 \pm 5.99$ & $47.8 \pm 4.89$ & $44.3 \pm 5.60$ \\
Total bilirubin $(\mathrm{mg} / \mathrm{dl})$ & $0.77 \pm 0.15$ & $0.91 \pm 0.12$ & $1.37 \pm 0.14$ \\
Platelet count $\left(\times 10^{4}\right)$ & $17.5 \pm 1.08$ & $11.9 \pm 0.88$ & $10.9 \pm 1.01$ \\
Prothrombin time $(\%$ of & $97.1 \pm 3.60$ & $88.4 \pm 2.93$ & $75.4 \pm 3.37$ \\
$\quad$ normal) & & & \\
Albumin $(\mathrm{g} / \mathrm{dl})$ & $4.03 \pm 0.14$ & $3.96 \pm 0.11$ & $3.50 \pm 0.13$ \\
\hline
\end{tabular}

The values are presented as the mean \pm standard deviation

were as follows: $\mathrm{F} 0$ in five patients $(9.8 \%), \mathrm{F} 1$ in nine patients $(17.6 \%), \mathrm{F} 2$ in 13 patients $(25.4 \%), \mathrm{F} 3$ in eight patients $(15.6 \%)$ and $\mathrm{F} 4$ in 16 patients $(31.3 \%)$. The patients were divided into three groups according to the grade of fibrosis: F0 or F1 as normal/mild fibrosis (F0/ 1 group; $n=14), \mathrm{F} 2$ or $\mathrm{F} 3$ as moderate/severe fibrosis (F2/ 3 group; $n=21$ ) and $\mathrm{F} 4$ as cirrhosis (F4 group; $n=16$ ). In the F4 group, all patients were classified as having a ChildPugh grade A status (Table 1).

\section{Statistical analysis}

All statistical analyses were conducted using the JMP software program Ver9 (SAS, Cary, NC), and a medical statistician reviewed all data. The patients were divided into three groups according to the Metavir score (F0-F1, F2-F3, F4). The data are expressed as the mean \pm standard deviation or median (interquartile range), as appropriate. Comparisons of the PVSG, HVAT, HV-HA interval time, HV-PV interval time and serum albumin levels were made using the Tukey-Kramer test. Comparisons of the HASG, HVSG, ICG-R15, HA and PT \% values were made nonparametrically using the Steel-Dwass test. Cirrhosis was defined as a Metavir score of F4. ROC analyses were conducted to assess the diagnostic value of each parameter for detecting cirrhosis. The optimal cutoff value of each parameter was determined according to the Youden index; that is, sensitivity + specificity -1 is maximized at the cutoff value. A Spearman rank correlation coefficient analysis was used to test for correlations between the PVSG and conventional biochemical markers. The strength of each correlation was expressed as $\rho$. The $\rho$ value was interpreted as follows: $0.7 \leq|\rho|=$ strong correlation; $0.4 \leq|\rho|<0.7=$ moderate correlation, $0.2 \leq|\rho|<0.4=$ weak correlation; $|\rho|<0.2=$ no correlation. A two-sided $p$ value of $<0.05$ was considered to be statistically significant.

\section{Results}

Sonazoid injection was well tolerated by all patients, and no adverse events were noted. The examinations were successfully performed in all patients.

Microbubble behavior in each vessel

In the patients with a normal liver, the microbubbles first reached the HA, then the PV and finally the HV. The HA and PV were both strongly enhanced. In the patients with cirrhosis, the microbubbles reached the $\mathrm{HV}$ earlier than that observed in the patients with a normal liver. In addition, the visual intensity of the PV was weak (Fig. 4). 

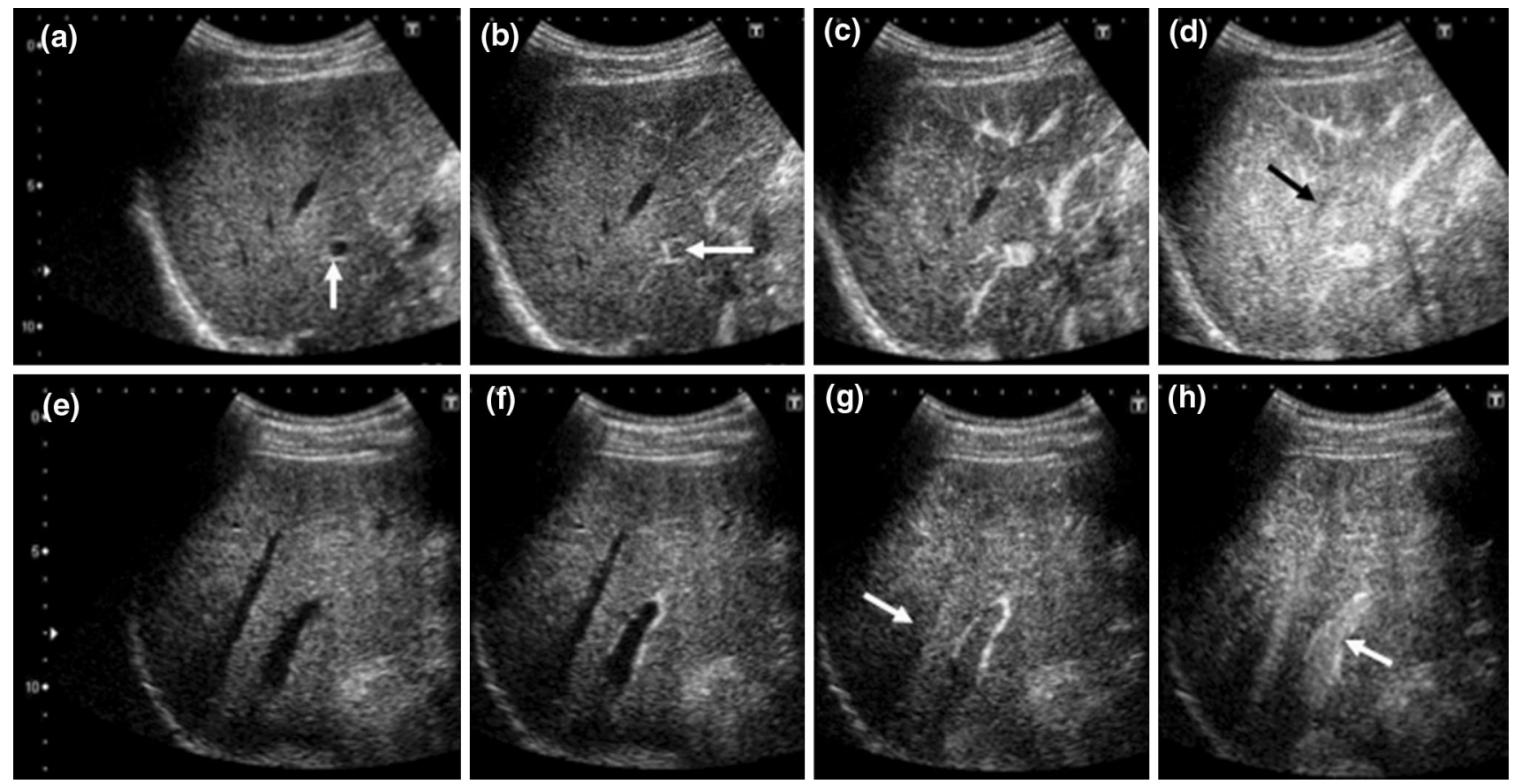

Fig. 4 Pulse-inversion imaging in the normal liver (a-d) and cirrhotic liver $(\mathbf{e}-\mathbf{h})$. In patients with a normal liver, the contrast agent arrives first in the HA (a arrow), then in the PV (b arrow) and finally in the HV (d black arrow). The HA and PV are both strongly enhanced $(\mathbf{b}, \mathbf{c})$. In patients with cirrhosis, the microbubbles reach the HV (g arrow) earlier than that observed in the patients with a normal liver. The intensity of the portal vein (h arrow) is weak compared with that observed in the normal liver

Table 2 Values of the slope gradients of the hepatic vessels, HVAT, interval times and conventional biochemical markers in each group

\begin{tabular}{llllll} 
F0/1 group $n=14$ & F2/3 group $n=21$ & F4 group $n=16$ & $p$ value & \\
\cline { 3 - 5 } & & F0/1 vs. F2/3 & F0/1 vs. F4 & F2/3 vs. F4
\end{tabular}

\begin{tabular}{|c|c|c|c|c|c|c|}
\hline \multicolumn{7}{|l|}{ Slope gradients } \\
\hline HASG & $24.7(10.9-49.1)$ & $22.4(15.4-29.8)$ & $20.2(18.2-28.9)$ & 0.8968 & 0.7835 & 0.8968 \\
\hline PVSG & $29.1 \pm 2.27$ & $23.1 \pm 1.86$ & $14.7 \pm 2.13$ & 0.0476 & $<0.0001$ & 0.0044 \\
\hline HVSG & $10.0(6.08-12.1)$ & $13.6(5.67-22.9)$ & $12.3(9.59-24.0)$ & 0.4261 & 0.5390 & 0.8533 \\
\hline \multicolumn{7}{|l|}{ HVAT and interval times } \\
\hline HVAT (s) & $31.5 \pm 1.81$ & $23.4 \pm 1.48$ & $27.3 \pm 1.69$ & 0.0030 & 0.2130 & 0.2010 \\
\hline HV-HA interval time (s) & $10.5 \pm 0.64$ & $7.56 \pm 0.52$ & $6.38 \pm 0.60$ & 0.0025 & $<0.0001$ & 0.3037 \\
\hline HV-PV interval time (s) & $6.45 \pm 0.68$ & $3.05 \pm 0.56$ & $1.82 \pm 0.64$ & 0.0010 & $<0.0001$ & 0.3202 \\
\hline \multicolumn{7}{|l|}{ Biochemical markers } \\
\hline ICG-15R (\%) & $17.3(12.3-24.0)$ & $22.2(10.3-37.5)$ & $45.1(30.8-67.5)$ & 0.4260 & 0.0003 & 0.0083 \\
\hline Hyaluronic acid (ng/ml) & $43.5(23.8-66.3)$ & $124(62.0-266)$ & $465(238-863)$ & 0.0023 & $<0.0001$ & 0.0083 \\
\hline Prothrombin time $(\%)$ & $98.5(87.5-103)$ & $87.0(82.5-96.5)$ & $71.5(58.3-94.0)$ & 0.1107 & 0.0088 & 0.0927 \\
\hline Albumin $(\mathrm{g} / \mathrm{dl})$ & $4.03 \pm 0.14$ & $3.95 \pm 0.11$ & $3.50 \pm 0.13$ & 0.9081 & 0.0176 & 0.0253 \\
\hline
\end{tabular}

The values are presented as the mean \pm standard deviation or median (interquartile range)

$H A S G$ hepatic artery slope gradient, $P V S G$ portal vein slope gradient, $H V S G$ hepatic vein slope gradient, $H V A T$ hepatic vein arrival time, $H V$ hepatic vein, $H A$ hepatic artery, $P V$ portal vein

Slope gradient

The SG of each vessel is shown in Table 2. The mean value of PVSG was $29.1 \pm 2.27$ in the F0/1 group, $23.1 \pm 1.86$ in the F2/3 group and $14.7 \pm 2.13$ in the F4 group. There were significant differences between the groups (F0/1 group vs. F2/3 group, $p=0.0476 ; \mathrm{F} 0 / 1$ group vs. F4 group, $p<0.0001 ; \mathrm{F} 2 / 3$ group vs. F4 group, $p=0.0044)$. No significant differences were observed in HASG or HVSG. 
HVAT, HV-HA interval time and HV-PV interval time

The HVAT, HV-HA interval time and HV-PV interval time values are shown in Table 2 . The mean value of HVAT was $31.5 \pm 1.81 \mathrm{~s}$ in the F0/1 group, $23.4 \pm 1.48 \mathrm{~s}$ in the F2/3 group and $27.3 \pm 1.69 \mathrm{~s}$ in the F4 group. There were significant differences between the F0/1 group and the $\mathrm{F} 2 / 3$ group $(p=0.0030)$; however, no differences were observed between the $\mathrm{F} 0 / 1$ and $\mathrm{F} 2 / 3$ groups $(p=0.2130)$ or the F2/3 and F4 groups $(p=0.2010)$. The mean values of the HV-HA interval time and the HV-PV interval time were $10.5 \pm 0.64$ and $6.45 \pm 0.68 \mathrm{~s}$, respectively, in the F0/1 group, $7.56 \pm 0.52$ and $3.05 \pm 0.56 \mathrm{~s}$, respectively, in the F2/3 group and $6.38 \pm 0.60$ and $1.82 \pm 0.64 \mathrm{~s}$, respectively, in the F4 group. For both parameters, there were significant differences between the F0/1 and F2/3 groups ( $p=0.0025, p=0.0010$, respectively) and the $\mathrm{F} 0 /$ 1 and F4 groups ( $p<0.0001, p<0.0001$, respectively); however, no differences were observed between the F2/3 and $\mathrm{F} 4$ groups ( $p=0.3037, p=0.3202$, respectively).

\section{Biochemical markers}

The values of the conventional biochemical markers ICG-R15, HA, PT \% and the serum albumin level are shown in Table 2. The median ICG-R15 value was $17.3 \%(12.3-24.0 \%)$ in the F0/1 group, $22.2 \%(10.3-37.5 \%)$ in the F2/3 group and $45.1 \%(30.8-67.5 \%)$ in the F4 group. There were significant differences between the $\mathrm{F} 0 / 1$ group and the $\mathrm{F} 4$ group $(p=0.0003)$ and between the F2/3 group and the F4 group $(p=0.0083)$; however, no differences were observed between the F0/1 and F2/3 groups $(p=0.4260)$. The median HA value was $43.5 \mathrm{ng} / \mathrm{ml}(23.8-66.3 \mathrm{ng} / \mathrm{ml})$ in the F0/1 group, $124 \mathrm{ng} /$ $\mathrm{ml}(62-266 \mathrm{ng} / \mathrm{ml})$ in the $\mathrm{F} 2 / 3$ group and $465 \mathrm{ng} / \mathrm{ml}$ $(238-863 \mathrm{ng} / \mathrm{ml})$ in the F4 group. All data for the HA showed significant differences between the groups (F0/1 vs. F2/3, $p=0.0023 ; \mathrm{F} 0 / 1$ vs. $\mathrm{F} 4, p<0.0001 ; \mathrm{F} 2 / 3$ vs. $\mathrm{F} 4, p=$ $0.0083)$. The median PT \% value was $98.5 \%(87.5-103 \%)$ in the F0/1 group, $87.0 \%(82.5-96.5 \%)$ in the F2/3 group and $71.5 \%$ (58.3-94.0 \%) in the F4 group. Only the F0/1 group and the F4 group differed significantly in this parameter $(p=0.0088)$. The mean serum albumin level was $4.03 \pm 0.14 \mathrm{~g} / \mathrm{dl}$ in the F0/1 group, $3.95 \pm 0.11 \mathrm{~g} / \mathrm{dl}$ in the F2/ 3 group and $3.50 \pm 0.13 \mathrm{~g} / \mathrm{dl}$ in the $\mathrm{F} 4$ group. There were significant differences between the F0/1 group and the F4 group ( $p=0.0176)$ and between the $\mathrm{F} 2 / 3$ group and the $\mathrm{F} 4$ group ( $p=0.0253$ ); however, no differences were observed between the F0/1 and F2/3 groups ( $p=0.9081$ ).

\section{Diagnostic accuracy}

The diagnostic accuracy of the PVSG, HVAT, HV-HA interval time, HV-PV interval time, ICG-R15, HA, PT \% and serum albumin level for detecting cirrhosis (Metavir $=$ F4) was analyzed using a ROC analysis. The area under the ROC curve (AUROC) for the PVSG, HVAT, HV-HA interval time and HV-PV interval time was $0.83571,0.54196,0.74018$ and 0.7623 , respectively (Fig. 5). The AUROC for the ICG-R15, HA, PT \% and serum albumin level was $0.84196,0.86161,0.75000$ and 0.78304 , respectively (Fig. 5). The results of the comparisons of the AUROCs for PVSG and the other parameters are shown in Table 3. The AUROC of PVSG was statistically different than that of HVAT; however, no differences were observed in the comparisons with other parameters (Table 3).

The optimal cutoff value for each parameter was determined according to the Youden Index (Table 4). The PVSG exhibited a sensitivity of $62.5 \%$, a specificity of $94.3 \%$ and an accuracy of $86.3 \%$.

Correlations between the PVSG and the biochemical markers

Scatter diagrams and the results of the correlation analyses of the PVSG and the ICG-R15, HA, PT \% and serum albumin level are shown in Fig. 6 and Table 5. The ICG-R15 and HA exhibited a moderate correlation with the PVSG with statistical differences $(\rho=-0.5691, p<0.0001$ for ICG-R15, $\rho=-0.4652, p=0.0006$ for HA). The PT $\%$ and the serum albumin level exhibited a weak correlation with the PVSG with statistical differences $(\rho=0.3015, \quad p=0.0315$ for PT $\%, \rho=0.3769, p=0.0064$ for the serum albumin level).

\section{Discussion}

In this study, we evaluated a new modality for diagnosing cirrhosis in comparison with conventional parameters. We observed that the PVSG of the TIC in the patients with compensated cirrhosis was significantly lower than that observed in the in noncirrhotic patients. When a PVSG cutoff value of 15 was used to diagnose cirrhosis, the specificity and accuracy were as high as 94.3 and $86.3 \%$, respectively. To our knowledge, this is the first report to demonstrate that measuring the PVSG using an ultrasound microbubble contrast agent can be used to discriminate patients with cirrhosis from those without. All of the patients in our study were candidates for surgical treatment. The patients in the F4 group had well-compensated cirrhosis, not advanced cirrhosis. In the diagnosis of wellcompensated cirrhosis, the analyses using the PVSG, which was calculated according to the combination of the signal intensity of the PV and the transit time of the contrast agent in the PV, exhibited higher accuracy than that observed in the transit time analyses only. 
(a) PVSG

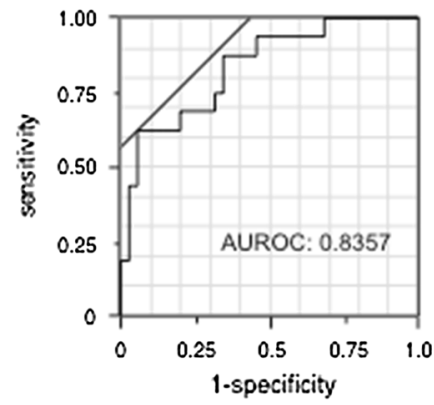

(e) ICG15R

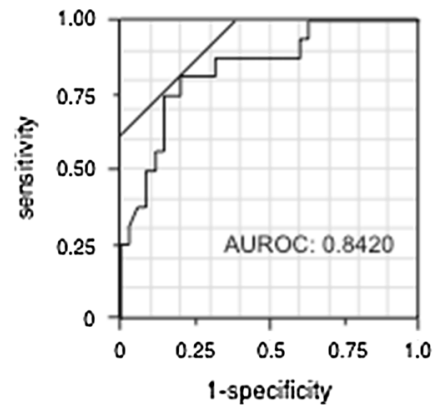

(b) HVAT

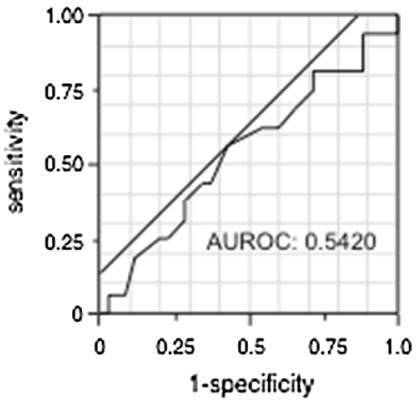

(f) Hyaluronic acid

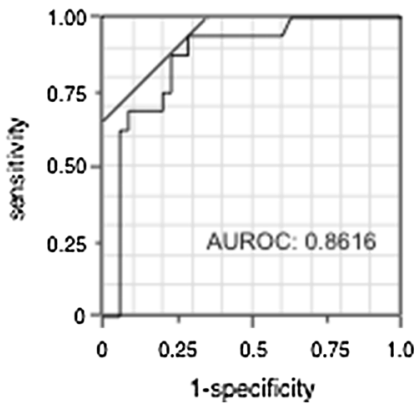

(c) HV-HA interval time

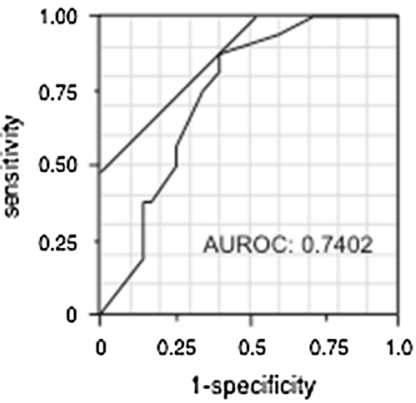

(g) Prothrombin time $(\%)$

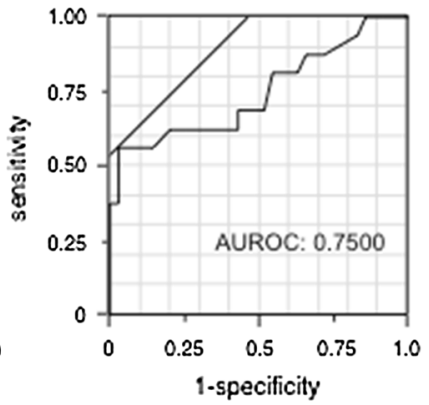

(d) HV.PV interval time

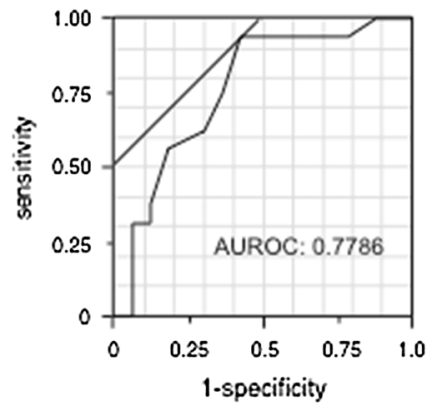

(h) Albumin

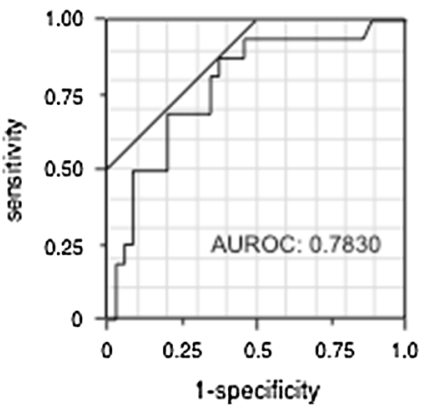

Fig. 5 ROC analysis of the PVSG (a), HVAT (b), HV-HA interval time (c), HV-PV interval time (d), ICG-R15 (e), hyaluronic acid level (f), prothrombin time $(\mathbf{g})$ and albumin level $(\mathbf{h})$ for the diagnosis of cirrhosis $($ Metavir $=$ F4)

Table 3 AUROC of each parameter and comparisons of the AUROC between the PVSG and the biochemical and CEUS parameters

\begin{tabular}{llll}
\hline Parameters & AUROC & $p$ vs. PVSG & $95 \%$ CI \\
\hline PVSG & 0.8357 & - & $0.6805-0.9240$ \\
HVAT & 0.5420 & 0.0039 & $0.3662-0.7079$ \\
HV-HA interval time & 0.7402 & 0.3278 & $0.5860-0.8515$ \\
HV-PV interval time & 0.7786 & 0.5248 & $0.6062-0.8788$ \\
ICG-15R & 0.8420 & 0.9319 & $0.6877-0.9280$ \\
Hyaluronic acid & 0.8616 & 0.7441 & $0.7142-0.9394$ \\
Prothrombin time (\%) & 0.7500 & 0.5551 & $0.5551-0.8782$ \\
Albumin & 0.7830 & 0.6159 & $0.6159-0.8904$
\end{tabular}

AUROC area under the receiver operating characteristic curve, $P V S G$ portal vein slope gradient, CEUS contrast-enhanced ultrasonography, $H V A T$ hepatic vein arrival time, $H V$ hepatic vein, $H A$ hepatic artery, CI confidence interval

Previous studies have shown that the HVAT, HV-HA interval and $\mathrm{HV}-\mathrm{PV}$ interval demonstrate high accuracy in diagnosing cirrhosis and can be used to predict the disease severity $[8,12]$. These measurements reflect intrahepatic arteriovenous and portovenous shunting caused by vascular remodeling at the sinusoidal level. However, in our study, the diagnostic accuracy of these parameters for diagnosing cirrhosis was lower than we had expected (Table 4) [8, 12]. One possible reason is that the contrast agent injection time may vary among patients. In this study, three different collaborators injected the contrast agent manually, with
Table 4 Sensitivity, specificity and accuracy of the PVSG, HVAT, interval times and conventional biochemical markers for diagnosing cirrhosis

\begin{tabular}{|c|c|c|c|c|}
\hline & $\begin{array}{l}\text { Cutoff } \\
\text { value }\end{array}$ & $\begin{array}{l}\text { Sensitivity } \\
(\%)\end{array}$ & $\begin{array}{l}\text { Specificity } \\
(\%)\end{array}$ & $\begin{array}{l}\text { Accuracy } \\
(\%)\end{array}$ \\
\hline PVSG & $<15$ & 62.5 & 94.3 & 86.3 \\
\hline HVAT (s) & $<28$ & 56.3 & 57.1 & 56.8 \\
\hline $\begin{array}{l}\text { HV-HA interval time } \\
\text { (s) }\end{array}$ & $<8.4$ & 87.5 & 60 & 68.6 \\
\hline $\begin{array}{l}\mathrm{HV}-\mathrm{PV} \text { interval time } \\
\text { (s) }\end{array}$ & $<4$ & 93.8 & 57.1 & 68.6 \\
\hline ICG-15R (\%) & $>30$ & 81.3 & 80 & 80.4 \\
\hline $\begin{array}{l}\text { Hyaluronic acid } \\
(\mathrm{ng} / \mathrm{ml})\end{array}$ & $>131$ & 93.8 & 71.4 & 78.4 \\
\hline Prothrombin time $(\%)$ & $<73$ & 56.2 & 97.1 & 84.3 \\
\hline Albumin $(\mathrm{g} / \mathrm{dl})$ & $<3.93$ & 87.5 & 62.9 & 70.6 \\
\hline
\end{tabular}

$P V S G$ portal vein slope gradient, HVAT hepatic vein arrival time, $H V$ hepatic vein, $H A$ hepatic artery, $P V$ portal vein

likely variation among injection times, ultimately affecting the HVAT. The HV-HA and HV-PV interval times are more accurate than the HVAT, as they are not affected by individual variations in injection times. However, in our results, the mean HV-HA interval time and HV-PV interval time were shorter than those previously reported [12]. The transit time of the contrast agent is reported to decrease in patients with liver tumors due to tumoral 
Fig. 6 Scatter diagram of the PVSG and the ICG-R15, hyaluronic acid level, prothrombin time and albumin level
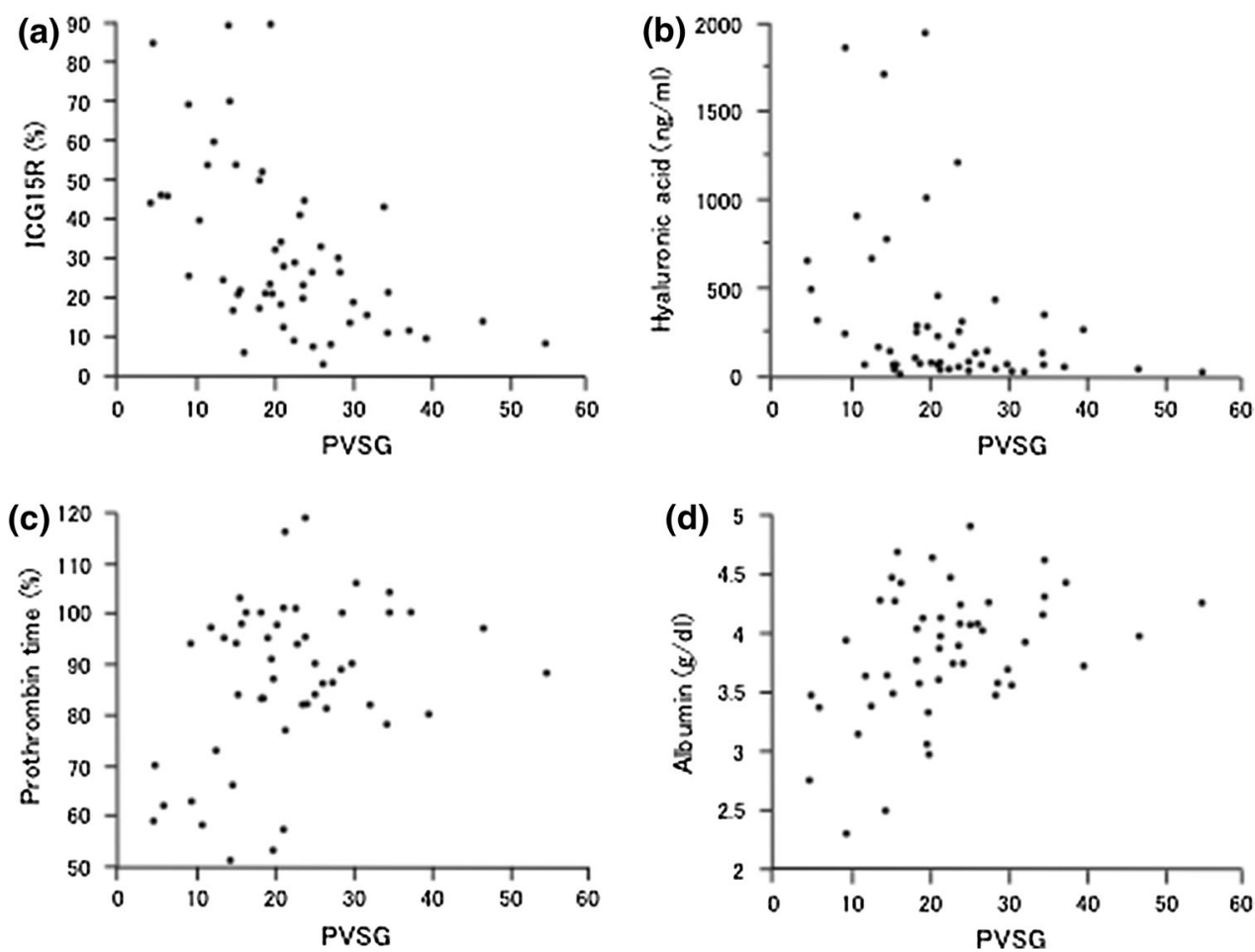

Table 5 Correlations between the PVSG and conventional biochemical markers

\begin{tabular}{lrr}
\hline Parameters & \multicolumn{1}{l}{$\rho$} & \multicolumn{1}{c}{$p$ value } \\
\hline ICG-15R & -0.5691 & $<0.0001$ \\
Hyaluronic acid & -0.4652 & 0.0006 \\
Prothrombin time (\%) & 0.3015 & 0.0315 \\
Albumin & 0.3769 & 0.0064 \\
\hline
\end{tabular}

$P V S G$ portal vein slope gradient

arteriovenous or portovenous shunting [18-20]. All of the patients in our study had liver tumors, which may have shortened the transit times, affecting the accuracy of diagnosing cirrhosis. Concerning this issue, in the clinical setting, many patients with cirrhosis have liver tumors, and the ability to detect well-compensated cirrhosis in these patients is critical for identifying surgical candidates. In this respect, determining the PVSG, which is not affected by tumoral intrahepatic shunting, is thought to be more useful than transit time analyses.

As for the intrahepatic arterial blood flow, it is well known that the hepatic arterial flow increases in patients with liver cirrhosis in order to compensate for the decreased PV blood flow due to the "hepatic arterial buffer response" [14]. Despite this phenomenon, our results revealed no significant differences in the HASG between the F4 group and the other groups. In reports of Doppler sonography, a high resistive index of the HA is observed in patients with severe cirrhosis; however, the HA flow remains normal in most cirrhotic patients [21, 22]. The subjects in this study were limited to those with wellcompensated cirrhosis, and our results showed that the HA flow was not dramatically changed in this group of patients.

In order to assess the clinical significance of the PVSG, we compared the AUROC of the PVSG with that of other diagnostic parameters. The AUROC of the PVSG was higher than that of the HVAT and interval times. Compared with the conventional biochemical parameters, the AUROCs of the ICG-R15 and HA were higher than that of the PVSG. As a result, the diagnostic impact of the PVSG was not superior to that of the ICG-R15 or HA. Despite this finding, the PVSG exhibited high specificity and accuracy in diagnosing cirrhosis (94.3 and $86.3 \%$, respectively). In addition, in the correlation analysis, the PVSG demonstrated moderate correlations with ICG-R15 and HA. In many patients, it is difficult to distinguish between those with and without cirrhosis using one parameter, especially patients with well-compensated cirrhosis. Our results emphasize that combination assays, including measurements of the PVSG and other parameters, such as the ICG$15 \mathrm{R}$ and HA, can be used to identify well-compensated cirrhosis patients more accurately.

There are some limitations to this study. First, this study was cross-sectional and involved different etiologies of liver disease, including HBV, HCV and alcoholic and cryptogenic hepatitis. From a pathologic standpoint, major differences have been reported between cirrhosis caused by hepatitis viruses and that caused by 
alcoholism, with a resultant difference in intrahepatic hemodynamics [23]. The smaller regenerative nodules observed in patients with alcoholic cirrhosis are more likely to cause venous compression and impede the early outflow, leading to portal hypertension. Furthermore, in cases of viral hepatitis, it is reported that certain histologic characteristics of $\mathrm{HCV}$ cirrhosis are distinct from those of $\mathrm{HBV}$ cirrhosis. Therefore, future studies should be composed of a cohort recruited from a homogeneous group of patients. A second limitation is that different microbubble contrast agents were used in prior studies on which we based our comparisons. The majority of reported studies used Levovist (Schering, Berlin, Germany), while other studies used Optison (Amersham Health, Milwaukee, WI, USA) [24], SonoVue (Bracco, Milan, Italy) [25] or Sonazoid (GE Healthcare, Oslo, Norway) [16]. These agents have different chemical properties. Levovist, a first-generation agent, is very fragile against acoustic pressure, while SonoVue and Sonazoid, second-generation agents, are more stable. Bloomly et al. [26] showed that Levovist and Sonazoid are taken up in the liver and spleen beyond the vascular phase, and Lim et al. [25, 27] demonstrated definitive uptake of SonoVue in the spleen with no substantial uptake in the liver. These differences could possibly result in different signal intensities and transit times. Considering these limits is important in functional examinations performed using microbubble contrast agents, and this issue should be clarified in future studies.

In conclusion, we have shown for the first time that the PVSG is a unique and reliable parameter with the potential to be a diagnostic tool for identifying surgical candidates among patients with well-compensated cirrhosis in combination with other conventional modalities. Although this technique requires further investigations, it is a promising useful tool for managing patients with chronic liver disease and conducting preoperative assessments of cirrhosis.

Conflict of interest Yuichi Goto and each of the co-authors have no conflicts of interest to disclose.

Open Access This article is distributed under the terms of the Creative Commons Attribution License which permits any use, distribution, and reproduction in any medium, provided the original author(s) and the source are credited.

\section{References}

1. Schuppan D, Afdhal NH. Liver cirrhosis. Lancet. 2008;371:838-51.

2. Iwao T, Toyonaga A, Oho K, Tayama C, Masumoto H, Sakai T, et al. Value of Doppler ultrasound parameters of portal vein and hepatic artery in the diagnosis of cirrhosis and portal hypertension. Am J Gastroenterol. 1997;92:1012-7.

3. Luo JC, Hwang SJ, Chang FY, Chu CW, Lai CR, Wang YJ, et al. Simple blood tests can predict compensated liver cirrhosis in patients with chronic hepatitis C. Hepatogastroenterology. 2002;49:478-81.

4. Sheth SG, Flamm SL, Gordon FD, Chopra S. AST/ALT ratio predicts cirrhosis in patients with chronic hepatitis $\mathrm{C}$ virus infection. Am J Gastroenterol. 1998;93:44-8.

5. Wai CT, Greenson JK, Fontana RJ, Kalbfleisch JD, Marrero JA, Conjeevaram HS, et al. A simple noninvasive index can predict both significant fibrosis and cirrhosis in patients with chronic hepatitis C. Hepatology. 2003;38:518-26.

6. Bravo AA, Sheth SG, Chopra S. Liver biopsy. N Engl J Med. 2001;344:495-500.

7. Saadeh S, Cammell G, Carey WD, Younossi Z, Barnes D, Easley $\mathrm{K}$. The role of liver biopsy in chronic hepatitis C. Hepatology. 2001;33:196-200.

8. Lim AK, Taylor-Robinson SD, Patel N, Eckersley RJ, Goldin $\mathrm{RD}$, Hamilton $\mathrm{G}$, et al. Hepatic vein transit times using a microbubble agent can predict disease severity non-invasively in patients with hepatitis C. Gut. 2005;54:128-33.

9. Blomley MJ, Lim AK, Harvey CJ, Patel N, Eckersley RJ, Basilico R, et al. Liver microbubble transit time compared with histology and Child-Pugh score in diffuse liver disease: a cross sectional study. Gut. 2003;52:1188-93.

10. Albrecht T, Blomley MJ, Cosgrove DO, Taylor-Robinson SD, Jayaram V, Eckersley R, et al. Non-invasive diagnosis of hepatic cirrhosis by transit-time analysis of an ultrasound contrast agent. Lancet. 1999;353:1579-83.

11. Searle J, Mendelson R, Zelesco M, Sanford J, Cheng W, McKinstry $\mathrm{C}$, et al. Non-invasive prediction of the degree of liver fibrosis in patients with hepatitis $\mathrm{C}$ using an ultrasound contrast agent. A pilot study. J Med Imaging Radiat Oncol. 2008;52:130-3.

12. Hirota M, Kaneko T, Sugimoto H, Kure S, Inoue S, Takeda S, et al. Intrahepatic circulatory time analysis of an ultrasound contrast agent in liver cirrhosis. Liver Int. 2005;25:337-42.

13. Sugimoto H, Kaneko T, Hirota M, Tezel E, Nakao A. Earlier hepatic vein transit-time measured by contrast ultrasonography reflects intrahepatic hemodynamic changes accompanying cirrhosis. J Hepatol. 2002;37:578-83.

14. Cichoz-Lach H, Celiński K, Słomka M, Kasztelan-Szczerbińska B. Pathophysiology of portal hypertension. J Physiol Pharmacol. 2008;59:231-8.

15. Ishibashi H, Maruyama H, Takahashi M, Fujiwara K, Imazeki F, Yokosuka O. Assessment of hepatic fibrosis by analysis of the dynamic behavior of microbubbles during contrast ultrasonography. Liver Int. 2010;30:1355-63.

16. Takahashi M, Maruyama H, Ishibashi H, Yoshikawa M, Yokosuka O. Contrast-enhanced ultrasound with perflubutane microbubble agent: evaluation of differentiation of hepatocellular carcinoma. AJR Am J Roentgenol. 2011;196:123-31.

17. Ishak K, Baptista A, Bianchi L, Callea F, De Groote J, Gudat F, et al. Histological grading and staging of chronic hepatitis. J Hepatol. 1995;22:696-9.

18. Bang N, Nielsen MB, Rasmussen AN, Osterhammel PA, Pedersen JF. Hepatic vein transit time of an ultrasound contrast agent: simplified procedure using pulse inversion imaging. Br J Radiol. 2001;74:752-5.

19. Zhou JH, Li AH, Cao LH, Jiang HH, Liu LZ, Pei XQ, et al. Haemodynamic parameters of the hepatic artery and vein can detect liver metastasis: assessment using contrast-enhanced ultrasound. Br J Radiol. 2008;81:113-9.

20. Zhang H, He Y, Du L, Wu Y. Shorter hepatic transient time can suggest coming metastases. J Ultrasound Med. 2010;29: 719-26.

21. Broide E, Farrant P, Reid F, Baker A, Meire H, Rela M, et al. Hepatic artery resistance index can predict early death in children with biliary atresia. Liver Transpl Surg. 1997;3:604-10. 
22. Vassiliades VG, Ostrow TD, Chezmar JL, Hertzler GL, Nelson RC. Hepatic arterial resistive indices: correlation with the severity of cirrhosis. Abdom Imaging. 1993;18:61-5.

23. Baggenstoss AH, Stauffer MH. Posthepatitic and alcoholic cirrhosis: clinicopathologic study of 43 cases of each. Gastroenterology. 1952;22:157-80.

24. Bernatik T, Strobel D, Häusler J, Hahn EG, Becker D. Hepatic transit time of an ultrasound echo enhancer indicating the presence of liver metastases-first clinical results. Ultraschall Med. 2002;23:91-5.

25. Lim AK, Patel N, Eckersley RJ, Goldin RD, Thomas HC, Cosgrove DO, et al. Hepatic vein transit time of SonoVue: a comparative study with Levovist. Radiology. 2006;240:130-5.
26. Blomley MJ, Albrecht T, Cosgrove DO, Eckersley RJ, ButlerBarnes J, Jayaram V, et al. Stimulated acoustic emission to image a late liver and spleen-specific phase of Levovist in normal volunteers and patients with and without liver disease. Ultrasound Med Biol. 1999;25:1341-52.

27. Lim AK, Patel N, Eckersley RJ, Taylor-Robinson SD, Cosgrove DO, Blomley MJ. Evidence for spleen-specific uptake of a microbubble contrast agent: a quantitative study in healthy volunteers. Radiology. 2004;231:785-8. 